\title{
A TEM Study on the Doping Behavior of Calcium in $\mathrm{LaCoO}_{3}$
}

\author{
Miaofang Chi, ${ }^{1}$ Siddhartha Pathak, ${ }^{2}$ Nigel Browning, ${ }^{1,3}$ Nina Orlovskaya ${ }^{4}$ \\ 1 University of California-Davis, Department of Chemical Engineering and Materials Science, \\ Davis, CA 95616, USA \\ 2 Drexel University, Department of Materials Engineering, 3200 Chestnut St., Philadelphia, PA \\ 19104, USA \\ 3 Lawrence Livermore National Laboratory, Materials Science and Technology Division, Livermore, \\ CA 94550 \\ 4 Michigan Technological University, Department of Materials Science and Engineering, 1400 \\ Townsend Drive, Houghton, MI 49931 USA
}

$\mathrm{LaCoO}_{3}$ based perovskites, which have attracted a lot of attention as potential candidates for cathode materials in solid oxide fuel cells (SOFC), also exhibit the unusual mechanical property of ferroelasticity. Ferroelasticity also allows the domains in the materials to switch orientations during mechanical loading [1]. As a result, crack growth is slowed down and the toughness of the materials is improved. Pure $\mathrm{LaCoO}_{3}$ has low conductivity and is not suitable for device applications. However, one effective way to improve its conductivity is the partial substitution of La with a lower valence element, which will introduce mixed ionic and electronic conductivity. Although the principles of this doping mechanism are straightforward, the distribution of dopants in $\mathrm{LaCoO}_{3}$ as well as their effect on the mechanical properties has rarely been investigated. The work presented here focuses primarily on the solubility evolution of calcium in $\mathrm{LaCoO}_{3}$, and its effect on the conductivity and mechanical properties of $\mathrm{LaCoO}_{3}$

$\mathrm{LaCoO}_{3}$ samples were doped with different concentrations of $\mathrm{Ca}$, including pure, $20 \%, 40 \%$ and $55 \%$ substitution on La. The microstructure of each sample was studied by Scanning Transmission Electron Microscopy (STEM). Electron energy loss spectroscopy (EELS) was performed to investigate the distribution of dopants and the valence states of cobalt.

Low-magnification STEM images of the samples are shown in Figure 1. These images were formed with a low collection angle in order to observe the domain structure, which is the feature of ferroelasticity at the micron-level. Figure 1 clearly shows that the domain structure is the main characteristic in the pure and $20 \%$ Ca doped samples. However, when the dopant amount increases to $40 \%$, the domain structure decreases significantly, and it disappears completely in the 55\% doped sample. This microstructure evolution indicates that the ferroelasticity diminishes and finally disappears with the dopant concentration ranged from $20 \%$ to $55 \%$. The contrast within the grains in the sample with $55 \% \mathrm{Ca}$ dopant results from the presence of a second phase, which was proved to be $\mathrm{CaCoO}_{3}$ by EELS. No precipitates or second phase were observed in the samples with lower doping levels. The relative atomic ratio of $\mathrm{Ca}$ to $(\mathrm{La}+\mathrm{Ca})$ from the grains and the grain boundaries in each sample is displayed in Figure 2. The Ca content in the grains of the sample with $40 \% \mathrm{Ca}$ is slightly higher than that in the $20 \%$ doped sample. However, the grain boundaries of the $40 \%$ doped sample contain much more $\mathrm{Ca}$ than the $20 \%$ sample. When the $\mathrm{Ca}$ doping amount increases to $55 \%$, the concentration of $\mathrm{Ca}$ remains the same in the grains, but it decreases on the grain boundaries. This compositional information has led to a clear picture of a dynamical equilibrium between grains, grain boundaries, and second phases. This solubility evolution is similar to that of Yittrium doped $\alpha$ 
-alumina [2]. An over-saturation of $\mathrm{Ca}$ on the grain boundaries appears to exist around $40 \%$ doping, where the system is in a metastable situation before the nucleation of $\mathrm{CaCoO}_{3}$. Further details on this doping mechanism and its effects on the microstructures of $\mathrm{LaCoO}_{3}$ will be presented. The mechanical properties and conductivities also will be correlated and discussed.

\section{References}

[1] E. K. H. Salije et al., Acta Cryst. A61 (2005) 3.

[2] C. M. Wang et al., Acta mater. 48 (2000) 2579.

[3] This research was performed at the National Center for Electron Microscopy and supported by the Department of Energy (DOE) under Contract No. DE-AC03-76SF00098 and by NSF under grant numbers DMR-0201770 and 0502765.

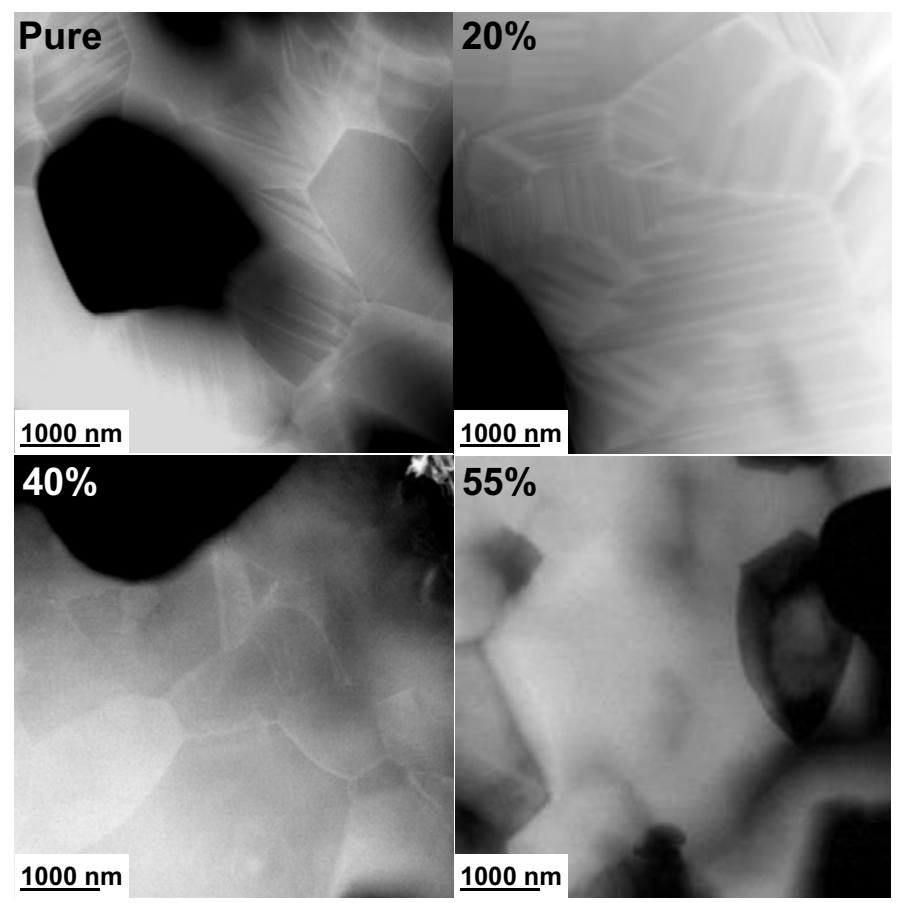

Fig. 1 STEM images of $\mathrm{LaCoO}_{3}$ with different $\mathrm{Ca}$ doping concentration.
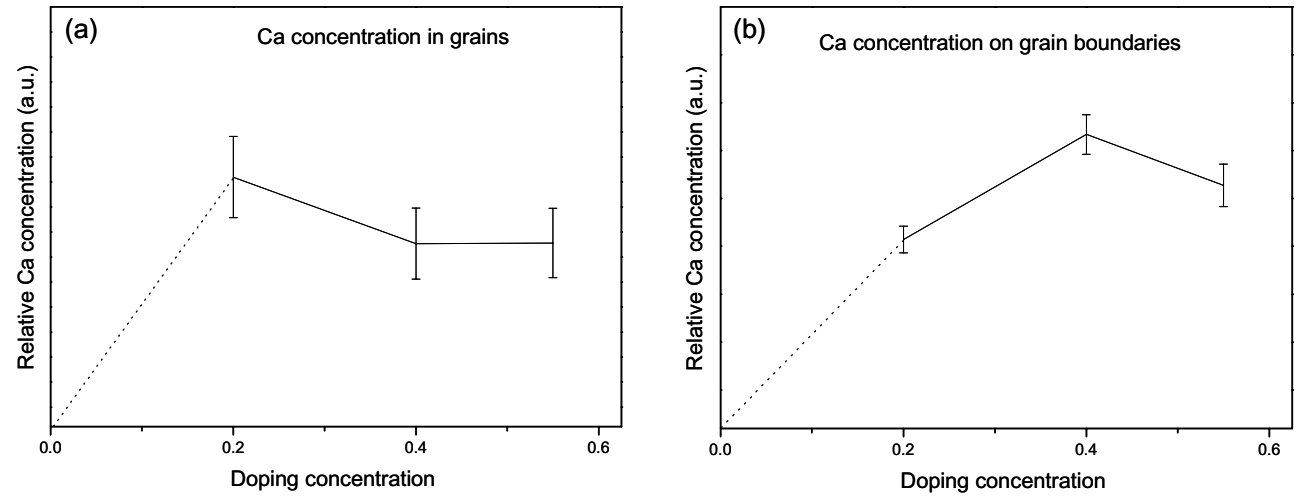

Fig 2 The calcium concentration in grains (a) and on grain boundaries (b). Each data point is an average of 20 spectra. The error bars are shown in the figures. 